\title{
Dinâmica dos micronutrientes em cafeeiros enxertados
}

\author{
André Dominghetti Ferreira ${ }^{1}$, Gladyston Rodrigues Carvalho ${ }^{2}$, Juliana Costa Rezende Abrahão ${ }^{2 *}$, \\ Ramiro Machado Rezende ${ }^{3}$, Cesar Elias Botelho ${ }^{2}$, Alex Mendonça de Carvalho ${ }^{4 *}$
}

\section{RESUMO}

As respostas à disponibilidade dos nutrientes variam entre espécies distintas dentro de um mesmo gênero, por causa, principalmente, das exigências nutricionais variáveis, capacidade de absorção, translocação e utilização dos nutrientes. O objetivo deste trabalho foi avaliar a eficiência de absorção, translocação e uso dos micronutrientes por diferentes cultivares de Coffea arabica L., enxertados em Apoatã IAC 2258 (Coffea canephora). O experimento foi instalado em casa de vegetação, utilizando-se o método de cultivo em solução nutritiva. Foi utilizado um fatorial 7 x 3 + 2, sendo sete cultivares de Coffea arabica L. (Palma II, Catucaí 2 SL, Oeiras MG 6851, Obatã IAC 1669-20, Acauã, Topázio MG 1190 e Paraíso MG H 419-1), três tipos de mudas (pé franco, autoenxertada e enxertada sobre o cultivar Apoatã IAC 2258) e duas testemunhas (Apoatã autoenxertado e Apoatã pé franco). O porta-enxerto utilizado influenciou negativamente na absorção de boro, ferro e manganês. A translocação dos micronutrientes boro e cobre obteve maiores índices nas mudas enxertadas. O cultivar Palma II, quando enxertado, apresentou o maior índice de utilização dos nutrientes, mostrando-se passível de ser enxertado. O porta-enxerto utilizado mostrou-se apto para a enxertia, por não sofrer influência negativa, tanto pela enxertia, quanto pelos cultivares utilizados.

Palavras-chave: Coffea arabica L, Coffea canephora, enxertia, nutrição.

\section{ABSTRACT}

\section{Dynamics of micronutrient in grafted coffee}

Responses to availability of nutrients vary between different species within the same genus, mainly because the different nutritional requirements, absorption capacity, translocation and use of nutrients, The purpose of this study was to evaluate the efficiency of absorption, translocation and use of micronutrients by different cultivars of Coffea arabica L. grafted into Apoatã IAC 2258 (Coffea canephora). The experiment was conducted in a greenhouse using cultivation in nutrient solution in a 7 x $3+2$ factorial design: seven cultivars of Coffea arabica L. (Palma II, Catucaí 2 SL, Oeiras MG 6851, Obatã IAC 1669-20, Acauã, Topázio MG 1190 and Paraíso MG H 419-1), three types of seedlings (ungrafted, self-grafted and grafted on cultivar Apoatã IAC 2258) and two controls (Apoatã self-grafted and Apoatã foot-free). The results showed that the rootstocknegatively influenced the absorption of boron, iron and manganese. The translocation of the micronutrients boron and copper had the highest rates in the grafted seedlings. The cultivar Palma II, when grafted, showed the highest rates of use of nutrients, showing that it can be grafted. The rootstock proved to be suitable for grafting, for not being negatively influenced by both the grafting and the cultivars used.

Key words: Coffea arabica L., Coffea canephora, grafting, nutrition.

\footnotetext{
Recebido para publicação em 30/12/2011 e aprovado em 21/02/2013.

${ }^{1}$ Engenheiro-Agrônomo, Doutor. Empresa Brasileira de Pesquisa Agropecuária - EMBRAPA, Av. Rádio Maia, 830, 79106-550, Campo Grande, Mato Grosso do Sul, Brasil. andre.dominghetti@embrapa.br (autor para correspondência).

${ }^{2}$ Engenheiro-Agrônomo, Doutor. Empresa de Pesquisa Agropecuária de Minas Gerais - EPAMIG, Caixa Postal 1176, 37200-000, Lavras, Minas Gerais, Brasil. carvalho@epamig.ufla.br; julianacosta@epamig.br; cesarbotelho@epamig.br

${ }^{3}$ Engenheiro-Agrônomo, Mestre. Departamento de Agricultura, Universidade Federal de Lavras, Caixa Postal 3037, 37200-000, Lavras, Minas Gerais, Brasil. ramiromr@globo.com ${ }^{4}$ Engenheiro-Agrônomo, Doutor. Departamento de Agricultura, Universidade Federal de Lavras, Caixa Postal 3037, 37200-000, Lavras, Minas Gerais, Brasil. carvalho.am@hotmail.com

* Bolsistas do Programa Nacional de Pós-doutorado - CNPq e CAPES
} 


\section{INTRODUÇÃO}

A utilização de diferentes espécies nas combinações de enxerto/porta-enxerto influencia, tanto na eficiência, quanto na exigência nutricional das plantas enxertadas. Dessa forma, as exigências nutricionais variáveis, a capacidade de absorção, de translocação e de utilização dos nutrientes nestas plantas resultam em diferentes índices de aproveitamento dos nutrientes.

Alguns fatores genéticos podem proporcionar diferenças nos teores foliares dos nutrientes, indicando que, entre cultivares e entre linhagens, existe maior ou menor eficiência de absorção, de translocação ou de utilização de nutrientes pela planta (Ferreira et al., 2010), havendo, portanto, a possibilidade de melhorar e, ou, selecionar cultivares mais eficientes quanto ao uso de nutrientes.

Acredita-se que, além de conferir resistência aos fitonematoides, a utilização do porta-enxerto na cafeicultura pode melhorar a eficiência no uso de nutrientes (Favarin et al., 2007), conferindo maior adaptabilidade às condições adversas de solo e áreas com precipitação pluviométrica limitada, por um sistema radicular mais desenvolvido e eficiente, aumentando com isso o potencial produtivo da planta enxertada.

Conhecer os efeitos e as possibilidades do processo de enxertia, em que o porta-enxerto possa aumentar a adaptabilidade da planta enxertada, é de grande relevância para a cafeicultura. Alguns trabalhos foram realizados com o objetivo de estudar a extração de nutrientes pelo cafeeiro, mas, com relação à enxertia, as pesquisas ainda são bastante escassas. Este trabalho objetivou avaliar, em cultivo hidropônico, a eficiência de absorção, translocação e uso dos micronutrientes por diferentes cultivares de Coffea arábica, enxertados em Apoatã IAC 2258 (Coffea canephora).

\section{MATERIAL E MÉTODOS}

$\mathrm{O}$ experimento foi instalado em casa de vegetação e conduzido por um período de 150 dias, utilizando-se o processo hidropônico de cultivo em vasos com solução nutritiva, contendo todos os nutrientes essenciais. O delineamento experimental utilizado foi o de blocos casualizados, no esquema fatorial 7 × $3+2$, sendo sete cultivares (Palma II, Catucaí 2SL, Oeiras MG 6851, Obatã IAC 1669-20, Acauã, Topázio MG 1190 e Paraíso MG H 419-1), três tipos de mudas (pé franco, auto-enxertadas e enxertadas sobre o cultivar Apoatã IAC 2258) e dois adicionais (Apoatã autoenxertado e Apoatã pé franco). Cada parcela experimental contou com uma planta, num total de cinco repetições.

A semeadura foi feita em caixas com areia lavada, realizando-se a enxertia quando as mudas atingiram o estádio de "palito de fósforo". O processo de enxertia utilizado foi do tipo hipocotiledonar. As plantas enxertadas, jun- tamente com as auto-enxertadas e as não enxertadas (pé franco), foram transplantadas para tubetes, contendo substrato comercial próprio para produção de mudas. Após o transplantio das mudas, elas foram mantidas em câmara de nebulização cobertas com sombrite $75 \%$ e com umidade de $90 \%$ para facilitar o pegamento das mudas enxertadas. Após 30 dias, as mudas foram levadas para o viveiro, onde permaneceram até atingirem cinco pares de folhas. Em seguida, as raízes foram lavadas, retirando-se todo o substrato, para que as mudas fossem colocadas em solução nutritiva de Hoagland \& Arnon (1950), com $20 \%$ da concentração recomendada, por um período de 30 dias, para a adaptação das mudas até o seu transplantio para os vasos definitivos, com capacidade volumétrica de dois litros.

À medida que ocorria a diminuição do volume da solução, por causa da transpiração, foi feita a reposição com água deionizada, até completar o volume original. Nos primeiros 30 dias após a fase de adaptação, a força da solução nutritiva (concentração dos nutrientes) foi de $30 \%$, do $31^{\circ}$ ao $90^{\circ}$ dia foi de $60 \%$, e do $91^{\circ}$ ao $150^{\circ}$ foi de $100 \%$ da concentração dos nutrientes. A solução foi trocada a cada quinze dias, visando a deixar as concentrações dos nutrientes próximas da ideal.

A avaliação do experimento foi realizada 150 dias após o transplantio para os vasos, separando-se a planta em raízes, caule e folhas. O material colhido foi lavado com água destilada, secado em estufa, com circulação forçada de ar, a $65^{\circ} \mathrm{C}$, por 168 horas, pesado e triturado em moinho tipo Wiley. Os teores de cobre, ferro, manganês e zinco presentes nos tecidos vegetais foram determinados por espectrofotometria de absorção atômica. Os teores de boro, após digestão por via seca (incineração), foram determinados por colorimetria (método da curcumina). Após a obtenção das concentrações dos nutrientes, calculouse o conteúdo em cada parte analisada (raiz, caule e folha). Para estudo da eficiência nutricional, foram utilizados os conceitos propostos pelos autores Siddiqi \& Glass (1981), Swiader et al. (1994) e Li et al. (1991).

As análises estatísticas foram realizadas, utilizandose o programa computacional Sisvar (Ferreira, 2008). Foi verificada a significância a $5 \%(\mathrm{p}<0,05)$ pelo teste F. Detectando-se diferenças significativas entre os tratamentos e entre as interações, foram feitos os desdobramentos e as médias foram comparadas entre si pelo teste de Tukey. As comparações entre fatorial e adicional foram analisadas pelo teste de Dunnett.

\section{RESULTADOS E DISCUSSÃO}

\section{Absorção radicular dos nutrientes}

A absorção do nutriente refere-se à entrada do elemento em forma iônica, ou molecular, nos espaços ou 
nas organelas intercelulares. Em relação a esta característica, as mudas autoenxertadas apresentaram o mesmo comportamento, ou superior, em relação à das mudas pé franco (Figura 1). Os tratamentos com mudas enxertadas apresentaram os menores valores para a absorção de nutrientes, podendo-se inferir que o sistema radicular da espécie $C$. canephora seja mais sensível à toxicidade desses elementos, apresentando níveis críticos de micronutrientes, nas folhas, bem menores do que os de C. arabica (Tomaz et al., 2006). De acordo com esses autores, fatores ligados à seletividade de seu sistema radicular não deixam que os micronutrientes atinjam concentrações prejudiciais à planta.

Quando se considera a média de eficiência de absorção do micronutriente, em relação a cada cultivar, observa-se que o cultivar Topázio MG 1190 mostrouse superior em absorção de boro, em relação aos cultivares Oeiras MG 6851, Obatã IAC 1669-20, Acauã e Palma II (Tabela 1). Da mesma forma, os cultivares Topázio MG 1190 e Paraíso MG H 419-1 apresentaram os maiores índices de absorção de Fe, enquanto os cultivares Oeiras MG 6851 e Acauã, os índices mais baixos. O cultivar Paraíso MG H 419-1 apresentou ainda maior absorção de zinco e os cultivares Palma II, Catuaí 2SL e Topázio apresentaram maior absorção de manganês. Possivelmente, a diferença detectada deuse em função das variações genéticas dos cultivares e morfológicas e fisiológicas do sistema radicular de cada cultivar, verificada também por outros autores (Caldeira et al., 2004, Tomaz et al., 2008, Mattiello et al., 2008). De acordo com Augusto et al. (2007), algumas espécies produzem extensivo sistema radicular e outras têm alta taxa de absorção por unidade de comprimento radicular, ou seja, alto influxo de nutrientes.

$\mathrm{Na}$ interação entre cultivares e tipos de mudas, não houve diferenças, pelo teste de F, nos teores de $\mathrm{B}, \mathrm{Cu}$ e Fe. Observa-se que a combinação Topázio MG 1190/ Apoatã IAC 2258 apresentou menor eficiência de absor- ção de zinco em relação ao respectivo pé franco, assim como Tomaz et al. (2006) encontraram maior eficiência de absorção de zinco, na combinação Catuaí Vermelho IAC 15/Conilon Muriaé 1, e diminuição, na combinação H41910-3-5-1/Apoatã IAC 2258. Os autores atribuiram este fato ao menor comprimento das raízes encontrado no Catuaí Vermelho IAC 15/Conilon Muriaé 1.

Observa-se, ainda, na Tabela 1, decréscimo no teor de manganês nas folhas das plantas enxertadas, em relação ao das não enxertadas, confirmando a seletividade do sistema radicular do Apoatã em absorver este nutriente, corroborando os resultados encontrados por Fahl et al. (1998), trabalhando com enxertia de $C$. arabica sobre $C$. canephora e sobre C. congensis.

\section{Translocação dos nutrientes na planta}

A translocação é a transferência do elemento de um órgão de absorção para outro qualquer. Para eficiência de translocação de boro, ferro, cobre e manganês entre cultivares; de boro e cobre entre tipos de mudas; e de boro, ferro e manganês na interação entre cultivares e tipos de mudas não houve diferença estatística.

A técnica da enxertia não prejudicou a translocação dos nutrientes (Figura 2). Houve, somente, redução de translocação para zinco e manganês, quando se compararam as mudas enxertadas em Apoatã, em relação às de autoenxertia e pé franco; houve, porém, uma maior taxa de translocação do micronutriente ferro em plantas enxertadas, em comparação com a de pé franco e de autoenxertadas. Da mesma forma, Soares et al. (2001) evidenciam um forte antagonismo na translocação de Zn e Fe em espécies de eucalipto. Lange et al. (2005) citam que a omissão de Mn pode aumentar a absorção de Fe, fato também observado neste trabalho.

De acordo com Natale et al. (2004), esse fenômeno de redução da translocação do $\mathrm{Zn}$ pode ser explicado pelo mecanismo das plantas de aumentarem a tolerância à toxidez desse nutriente, pois, nessas condições, tem-se

Médias de eficiência de absorção

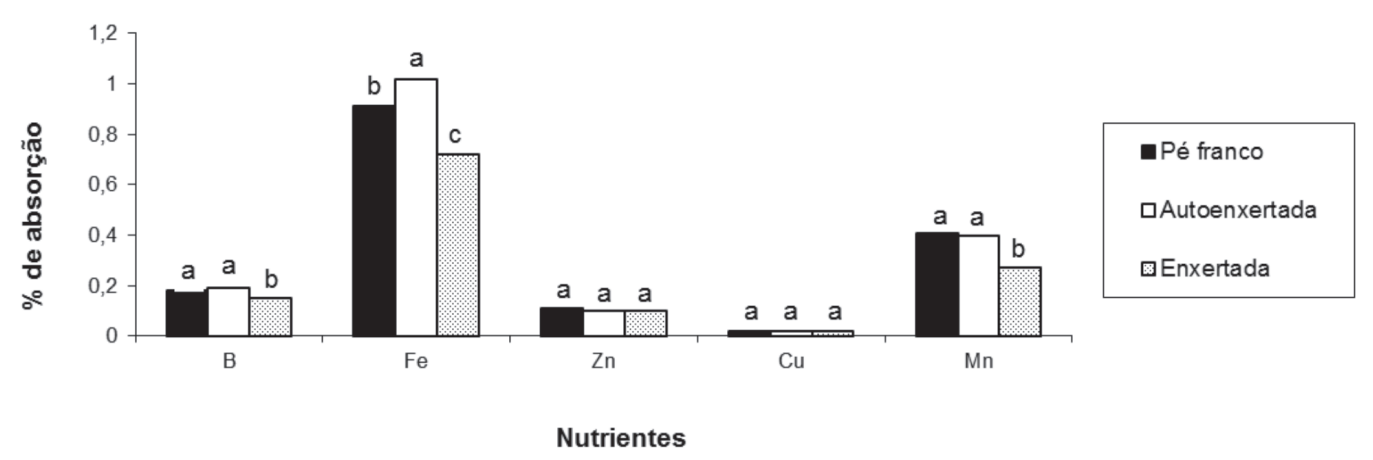

Figura 1. Eficiência de absorção dos nutrientes em mudas de café pé franco, auto-enxertada e enxertada. Médias seguidas pela mesma letra não diferem entre si a $5 \%(\mathrm{p}<0,05)$ pelo teste de Tukey.

Rev. Ceres, Viçosa, v. 60, n.2, p. 262-269, mar/abr, 2013 
maior acúmulo do mesmo nutriente nos vacúolos das células do córtex da raiz. A translocação de manganês comportou-se da mesma maneira que a absorção, ou seja, as mudas enxertadas foram menos eficientes que de pé franco, resultados estes coincidentes com os de Tomaz et al.
(2006), que encontraram menor eficiência de translocação do nutriente em questão quando as plantas de $C$. arabica foram enxertadas no C. canephora Apoatã IAC 2258.

Quando se consideram os valores médios de eficiência de translocação dos nutrientes para cada cultivar (Ta-

\section{Médias de eficiência de translocação}

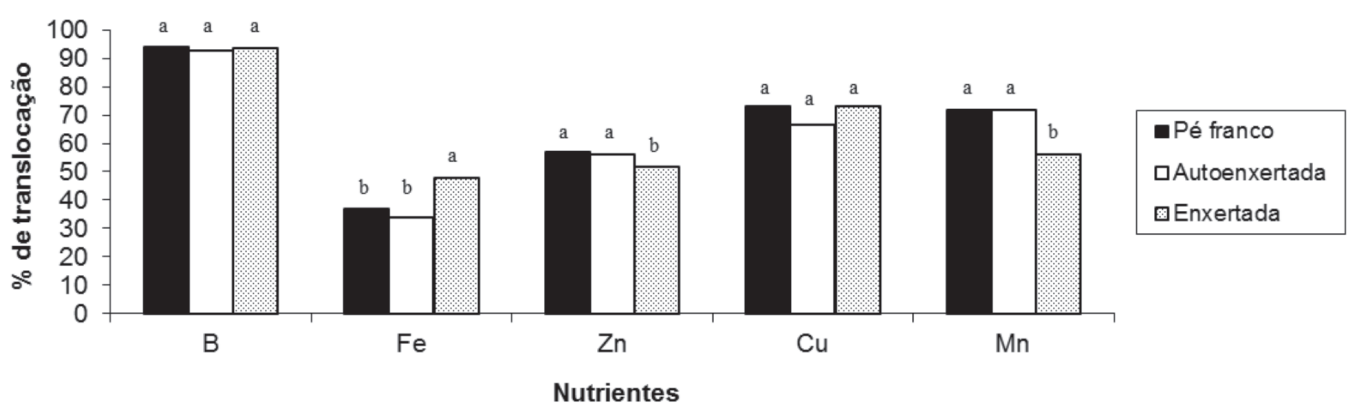

Figura 2. Eficiência de translocação dos nutrientes em mudas de café pé franco, auto- enxertada e enxertada. Médias seguidas pela mesma letra não diferem entre si a $5 \%(\mathrm{p}<0,05)$ pelo teste de Tukey.

Tabela 1. Eficiência média de absorção dos nutrientes no desdobramento de tipo de muda dentro de cultivar

\begin{tabular}{|c|c|c|c|c|c|}
\hline \multirow{2}{*}{ Tratamento } & B & $\mathbf{F e}$ & $\mathbf{Z n}$ & $\mathrm{Cu}$ & Mn \\
\hline & \multicolumn{5}{|c|}{$\mathrm{mg.g}^{-1}$} \\
\hline \multicolumn{6}{|l|}{ Palma II } \\
\hline pé franco & $0,16 \mathrm{a}$ & $0,87 \mathrm{a}$ & $0,10 \mathrm{a}$ & $0,02 \mathrm{a}$ & $0,41 \mathrm{a}$ \\
\hline autoenxertado & $0,17 \mathrm{a}$ & $1,15 \mathrm{a}$ & $0,11 \mathrm{a}$ & $0,02 \mathrm{a}$ & $0,47 \mathrm{a}$ \\
\hline Enxertado & $0,17 \mathrm{a}$ & $0,74 \mathrm{a}$ & $0,10 \mathrm{a}$ & $0,02 \mathrm{a}$ & $0,30 \mathrm{~b}$ \\
\hline \multicolumn{6}{|l|}{ Catucaí 2SL } \\
\hline pé franco & $0,21 \mathrm{a}$ & $1,00 \mathrm{a}$ & $0,11 \mathrm{a}$ & $0,02 \mathrm{a}$ & $0,53 \mathrm{a}$ \\
\hline autoenxertado & $0,21 \mathrm{a}$ & $1,03 \mathrm{a}$ & $0,10 \mathrm{a}$ & $0,02 \mathrm{a}$ & $0,47 \mathrm{a}$ \\
\hline Enxertado & $0,15 \mathrm{a}$ & $0,78 \mathrm{a}$ & $0,10 \mathrm{a}$ & $0,02 \mathrm{a}$ & $0,25 \mathrm{~b}$ \\
\hline \multicolumn{6}{|l|}{ Oeiras MG 6851} \\
\hline pé franco & $0,16 \mathrm{a}$ & $0,74 \mathrm{a}$ & $0,10 \mathrm{a}$ & $0,02 \mathrm{a}$ & $0,34 \mathrm{ab}$ \\
\hline autoenxertado & $0,16 \mathrm{a}$ & $0,90 \mathrm{a}$ & $0,10 \mathrm{a}$ & $0,01 \mathrm{a}$ & $0,35 \mathrm{a}$ \\
\hline Enxertado & $0,13 \mathrm{a}$ & $0,60 \mathrm{a}$ & $0,11 \mathrm{a}$ & $0,01 \mathrm{a}$ & $0,24 \mathrm{~b}$ \\
\hline \multicolumn{6}{|l|}{ Obatã IAC 1669-20 } \\
\hline pé franco & $0,17 \mathrm{a}$ & $0,89 \mathrm{a}$ & $0,07 \mathrm{a}$ & $0,02 \mathrm{a}$ & $0,27 \mathrm{a}$ \\
\hline autoenxertado & $0,17 \mathrm{a}$ & $0,92 \mathrm{a}$ & $0,08 \mathrm{a}$ & $0,02 \mathrm{a}$ & $0,34 \mathrm{a}$ \\
\hline Enxertado & $0,15 \mathrm{a}$ & $0,72 \mathrm{a}$ & $0,10 \mathrm{a}$ & $0,01 \mathrm{a}$ & $0,29 a$ \\
\hline \multicolumn{6}{|l|}{$\overline{\text { Acauã }}$} \\
\hline pé franco & $0,17 \mathrm{a}$ & $0,80 \mathrm{a}$ & $0,08 \mathrm{a}$ & $0,13 \mathrm{a}$ & $0,43 \mathrm{a}$ \\
\hline autoenxertado & $0,17 \mathrm{a}$ & $0,83 \mathrm{a}$ & $0,10 \mathrm{a}$ & $0,13 \mathrm{a}$ & $0,32 \mathrm{~b}$ \\
\hline Enxertado & $0,14 \mathrm{a}$ & $0,62 \mathrm{a}$ & $0,11 \mathrm{a}$ & $0,13 \mathrm{a}$ & $0,31 \mathrm{~b}$ \\
\hline \multicolumn{6}{|l|}{ Topázio MG 1190} \\
\hline pé franco & $0,21 \mathrm{a}$ & $1,10 \mathrm{a}$ & $0,14 \mathrm{a}$ & $0,02 \mathrm{a}$ & $0,52 \mathrm{a}$ \\
\hline autoenxertado & $0,23 \mathrm{a}$ & $1,14 \mathrm{a}$ & $0,10 \mathrm{~b}$ & $0,01 \mathrm{a}$ & $0,47 \mathrm{a}$ \\
\hline Enxertado & $0,15 \mathrm{a}$ & $0,74 \mathrm{a}$ & $0,08 \mathrm{~b}$ & $0,01 \mathrm{a}$ & $0,20 \mathrm{~b}$ \\
\hline \multicolumn{6}{|l|}{ Paraíso MG H 419-1 } \\
\hline pé franco & $0,18 \mathrm{a}$ & $0,95 \mathrm{a}$ & $0,14 \mathrm{a}$ & $0,02 \mathrm{a}$ & $0,40 \mathrm{a}$ \\
\hline autoenxertado & $0,18 \mathrm{a}$ & $1,17 \mathrm{a}$ & $0,11 \mathrm{a}$ & $0,02 \mathrm{a}$ & $0,38 \mathrm{a}$ \\
\hline Enxertado & $0,16 \mathrm{a}$ & $0,82 \mathrm{a}$ & $0,12 \mathrm{a}$ & $0,02 \mathrm{a}$ & $0,30 \mathrm{a}$ \\
\hline $\mathrm{CV}(\%)$ & 14,60 & 17,32 & 19,12 & 28,59 & 16,26 \\
\hline
\end{tabular}

Médias seguidas pela mesma letra na vertical - dentro de cada cultivar - não diferem entre si a $5 \%(\mathrm{p}<0,05)$ pelo teste de Tukey. 
bela 2), nota-se que a eficiência de translocação de zinco, no cultivar Catucaí 2SL, iguala-se apenas à de Acauã. A translocação de zinco, apresentada pelo cultivar Catucaí 2SL, evidencia o potencial desse cultivar para cultivo em solos com baixo teor desse nutriente, uma vez que apresentou eficiência de absorção intermediária à das demais, porém elevada capacidade de translocação. Resultado semelhante também foi obtido por Augusto et al. (2007), que encontraram variações entre os cultivares estudados quanto à translocação de zinco.

Para Marschner (1995), as diferenças genotípicas na eficiência nutricional podem estar relacionadas com a demanda celular de nutrientes, compartimentalização, utilização na parte aérea, no transporte a curta e a longa distância, na afinidade do sistema de absorção (km), concentração mínima (Cmin) e modificações na rizosfera.

Observando-se o desdobramento de tipos de mudas dentro de cultivar, nota-se que o cultivar Oeiras MG 6851, quando autoenxertado, apresentou menor eficiência de translocação de boro e cobre. O cultivar Acauã teve o mesmo comportamento em relação ao cobre. A movimentação de íons através das raízes e seu descarregamento no xilema envolvem vários mecanismos que podem limitar sua ascensão para a parte aérea e podem constituir diferenças genotípicas na absorção e movimentação dos nutrientes (Gerloff \& Gabelman, 1983). Dessa forma, a técnica da enxertia funcionou como um mecanismo limitante para a translocação de cobre, nos cultivares Acauã e Oeiras MG 6851 quando autoenxertados. Porém, esta limitação foi superada, quando se utilizou o porta-enxerto Apoatã IAC 2258, uma vez que mudas, enxertadas, ou não, apresentaram a mesma eficiência de translocação daquele nutriente. Os dados apresentados corroboram os encontrados por Tomaz et al. (2006), que verificaram aumentos de até $37,2 \%$ na eficiência de translocação de cobre, nas mudas enxertadas em relação à das suas respectivas mudas não enxertadas.

Tabela 2. Médias de eficiência de translocação dos nutrientes no desdobramento de tipo de muda dentro de cultivar

\begin{tabular}{|c|c|c|c|c|c|}
\hline \multirow{2}{*}{ Tratamento } & B & $\mathbf{F e}$ & Zn & $\mathrm{Cu}$ & Mn \\
\hline & \multicolumn{5}{|c|}{$\%$} \\
\hline \multicolumn{6}{|l|}{ Palma II } \\
\hline Pé franco & $93,63 \mathrm{a}$ & $36,90 \mathrm{a}$ & $51,00 \mathrm{a}$ & 72,77 a & $65,28 \mathrm{a}$ \\
\hline Autoenxertado & $94,24 \mathrm{a}$ & $32,67 \mathrm{a}$ & $54,81 \mathrm{a}$ & $65,23 \mathrm{a}$ & $74,05 \mathrm{a}$ \\
\hline Enxertado & $94,90 \mathrm{a}$ & $47,81 \mathrm{a}$ & 55,19 a & $69,51 \mathrm{a}$ & $45,53 \mathrm{a}$ \\
\hline \multicolumn{6}{|l|}{ Catucaí 2SL } \\
\hline Pé franco & $94,56 \mathrm{a}$ & 33,61 a & $65,56 \mathrm{a}$ & 68,77 a & $72,27 \mathrm{a}$ \\
\hline Autoenxertado & $93,84 \mathrm{a}$ & $36,33 \mathrm{a}$ & $67,64 \mathrm{a}$ & $68,66 \mathrm{a}$ & $72,67 \mathrm{a}$ \\
\hline Enxertado & $94,00 \mathrm{a}$ & 50,66 a & 60,96 a & 77,18 a & $68,26 \mathrm{a}$ \\
\hline \multicolumn{6}{|l|}{ Oeiras MG 6851} \\
\hline Pé franco & 94,07 a & 37,49 a & 56,09 a & 77,18 a & $71,55 \mathrm{a}$ \\
\hline Autoenxertado & $88,84 \mathrm{~b}$ & $30,67 \mathrm{a}$ & $49,60 \mathrm{a}$ & $64,76 \mathrm{~b}$ & $63,37 \mathrm{a}$ \\
\hline Enxertado & $93,01 \mathrm{a}$ & $49,67 \mathrm{a}$ & $45,23 \mathrm{a}$ & $77,12 \mathrm{a}$ & $50,87 \mathrm{a}$ \\
\hline \multicolumn{6}{|l|}{ Obatã IAC 1669-20 } \\
\hline Pé franco & $93,59 \mathrm{a}$ & $34,57 \mathrm{a}$ & $55,62 \mathrm{a}$ & $77,51 \mathrm{a}$ & $59,58 \mathrm{a}$ \\
\hline Autoenxertado & $92,70 \mathrm{a}$ & 35,66 a & $56,37 \mathrm{a}$ & 74,87 a & $68,85 \mathrm{a}$ \\
\hline Enxertado & $91,49 \mathrm{a}$ & 38,76 a & $50,27 \mathrm{a}$ & $67,02 \mathrm{a}$ & $44,59 \mathrm{a}$ \\
\hline \multicolumn{6}{|l|}{$\overline{\text { Acauã }}$} \\
\hline Pé franco & $93,68 \mathrm{a}$ & $43,30 \mathrm{a}$ & $69,85 \mathrm{a}$ & $69,63 \mathrm{a}$ & $83,33 \mathrm{a}$ \\
\hline Autoenxertado & $92,50 \mathrm{a}$ & $33,60 \mathrm{a}$ & $61,22 \mathrm{a}$ & $48,78 \mathrm{~b}$ & $79,03 \mathrm{a}$ \\
\hline Enxertado & $93,44 \mathrm{a}$ & 56,57 a & $46,26 \mathrm{~b}$ & 69,76 a & $63,76 \mathrm{a}$ \\
\hline \multicolumn{6}{|l|}{ Topázio MG 1190} \\
\hline Pé franco & $94,66 \mathrm{a}$ & $35,25 \mathrm{a}$ & 54,16 a & $70,56 \mathrm{a}$ & $79,37 \mathrm{a}$ \\
\hline Autoenxertado & $94,03 \mathrm{a}$ & $34,89 \mathrm{a}$ & $58,43 \mathrm{a}$ & $69,83 \mathrm{a}$ & $78,43 \mathrm{a}$ \\
\hline Enxertado & $92,25 \mathrm{a}$ & 44,93 a & 55,86 a & 75,88 a & $62,63 \mathrm{a}$ \\
\hline \multicolumn{6}{|l|}{ Paraíso MG H 419-1 } \\
\hline Pé franco & $93,31 \mathrm{a}$ & 38,39 a & $46,64 \mathrm{a}$ & $74,01 \mathrm{a}$ & $71,07 \mathrm{a}$ \\
\hline Autoenxertado & $92,51 \mathrm{a}$ & $33,13 \mathrm{a}$ & $44,61 \mathrm{a}$ & $74,52 \mathrm{a}$ & $65,43 \mathrm{a}$ \\
\hline Enxertado & 94,19 a & 47,29 a & $47,64 \mathrm{a}$ & 74,94 a & $58,07 \mathrm{a}$ \\
\hline $\mathrm{CV}(\%)$ & 1,57 & 11,14 & 15,90 & 8,96 & 12,69 \\
\hline
\end{tabular}

Médias seguidas pela mesma letra na vertical - dentro de cada cultivar - não diferem entre si a 5\% $(\mathrm{p}<0,05)$ pelo teste de Tukey. 


\section{Eficiência de uso dos nutrientes}

Analisando-se a média de eficiência de utilização dos nutrientes de cada cultivar, verifica-se que não houve interação significativa, pelo teste de F. Considerando-se as interações, observa-se que, para os micronutrientes boro, ferro e manganês, as plantas enxertadas apresentaram eficiência de uso igual ou superior à das não enxertadas (Tabela 3). Tomaz et al. (2006) também encontraram maior eficiência de uso de manganês nos cultivares Catuaí Vermelho IAC 15 e na progênie H514-5-5-3, quando enxertados em Apoatã IAC 2258.

Analisando-se a eficiência de uso do manganês pelos cultivares, quando em pé franco, verifica-se que os cultivares estudados, com exceção de Obatã IAC 1669-20 e Acauã foram beneficiados pela enxertia testada. De acordo com Willson (1987), plantas da espécie de C. canephora são mais sensíveis ao Mn do que as de C. arabica.

Em relação aos demais nutrientes, observa-se uma variação no comportamento dos cultivares, contudo, so- mente o cultivar Obatã IAC 1669-20 esteve, de uma maneira geral, entre os melhores para todos os nutrientes. Quanto às mudas autoenxertadas, o cultivar que mais se destacou foi ‘Topázio MG 1190', uma vez que apresentou os maiores índices de eficiência de uso para todos os nutrientes estudados. Já para mudas enxertadas, a maior eficiência de uso foi encontrada para 'Palma II'. Vários autores detectaram na cultura do cafeeiro diferenças na utilização de nutrientes pela planta, proporcionada por fatores genéticos, indicando que entre cultivares e entre linhagem existem diferenças nas concentrações foliares dos nutrientes (Tomaz et al., 2003; Augusto et al., 2007).

\section{Eficiência de absorção, translocação e de utilização dos adicionais}

A utilização dos tratamentos adicionais objetivou comparar o desenvolvimento do porta-enxerto, quando em pé franco e autoenxertado, com os demais tratamentos do fatorial, identificando qualquer influência da técnica da

Tabela 3. Eficiência média do uso dos micronutrientes no desdobramento de tipo de muda dentro de cultivar

\begin{tabular}{|c|c|c|c|c|c|}
\hline \multirow{2}{*}{ Tratamento } & B & $\mathbf{F e}$ & Zn & $\mathbf{C u}$ & Mn \\
\hline & \multicolumn{5}{|c|}{$\mathrm{g}^{2} \cdot \mathrm{mg}^{-1}$} \\
\hline \multicolumn{6}{|l|}{ Palma II } \\
\hline Pé franco & $0,51 \mathrm{a}$ & $0,09 \mathrm{a}$ & $0,85 \mathrm{a}$ & $4,60 \mathrm{a}$ & $0,20 \mathrm{~b}$ \\
\hline Autoenxertado & $0,54 \mathrm{a}$ & $0,08 \mathrm{~b}$ & $0,84 \mathrm{a}$ & $5,10 \mathrm{a}$ & $0,20 \mathrm{~b}$ \\
\hline Enxertado & $0,54 \mathrm{a}$ & $0,13 \mathrm{a}$ & $0,94 \mathrm{a}$ & $5,50 \mathrm{a}$ & $0,30 \mathrm{a}$ \\
\hline \multicolumn{6}{|l|}{ Catucaí 2SL } \\
\hline Pé franco & $0,33 \mathrm{a}$ & $0,07 \mathrm{a}$ & $0,61 \mathrm{~b}$ & $4,19 \mathrm{ab}$ & $0,13 \mathrm{~b}$ \\
\hline Autoenxertado & $0,41 \mathrm{a}$ & $0,09 \mathrm{a}$ & $0,85 \mathrm{a}$ & $5,12 \mathrm{a}$ & $0,18 \mathrm{ab}$ \\
\hline Enxertado & $0,36 \mathrm{a}$ & $0,07 \mathrm{a}$ & $0,56 \mathrm{~b}$ & $2,89 \mathrm{~b}$ & $0,23 \mathrm{a}$ \\
\hline \multicolumn{6}{|l|}{ Oeiras MG 6851} \\
\hline Pé franco & $0,45 \mathrm{~b}$ & $0,09 \mathrm{ab}$ & $0,69 \mathrm{a}$ & $4,03 \mathrm{a}$ & $0 ., 1 \mathrm{~b}$ \\
\hline Autoenxertado & $0,46 \mathrm{~b}$ & $0,08 \mathrm{~b}$ & $0,64 \mathrm{a}$ & $5,55 \mathrm{a}$ & $0,20 \mathrm{~b}$ \\
\hline Enxertado & $0,55 \mathrm{a}$ & $0,12 \mathrm{a}$ & $0,70 \mathrm{a}$ & $5,02 \mathrm{a}$ & $0,32 \mathrm{a}$ \\
\hline \multicolumn{6}{|l|}{ Obatã IAC 1669-20 } \\
\hline Pé franco & $0,49 \mathrm{a}$ & $0,09 \mathrm{a}$ & $1,19 \mathrm{a}$ & $4,74 \mathrm{a}$ & $0,31 \mathrm{a}$ \\
\hline Autoenxertado & $0,51 \mathrm{a}$ & $0,10 \mathrm{a}$ & $1,09 \mathrm{a}$ & $4,27 \mathrm{a}$ & $0,26 \mathrm{a}$ \\
\hline Enxertado & $0,46 \mathrm{a}$ & $0,10 \mathrm{a}$ & $0,71 \mathrm{~b}$ & $4,99 \mathrm{a}$ & $0,25 \mathrm{a}$ \\
\hline \multicolumn{6}{|l|}{$\overline{\text { Acauã }}$} \\
\hline Pé franco & $0,46 \mathrm{a}$ & $0,10 \mathrm{a}$ & $0,99 \mathrm{a}$ & $5,87 \mathrm{a}$ & $0,18 \mathrm{a}$ \\
\hline Autoenxertado & $0,51 \mathrm{a}$ & $0,10 \mathrm{a}$ & $0,89 \mathrm{a}$ & $5,62 \mathrm{a}$ & $0,26 \mathrm{a}$ \\
\hline Enxertado & $0,46 \mathrm{a}$ & $0,11 \mathrm{a}$ & $0,63 \mathrm{~b}$ & $5,87 \mathrm{a}$ & $0,22 \mathrm{a}$ \\
\hline \multicolumn{6}{|l|}{ Topázio MG 1190} \\
\hline Pé franco & $0,45 \mathrm{a}$ & $0,09 \mathrm{ab}$ & $0,67 \mathrm{~b}$ & $6,01 \mathrm{~b}$ & $0,18 \mathrm{~b}$ \\
\hline Autoenxertado & $0,52 \mathrm{a}$ & $0,11 \mathrm{a}$ & $1,14 \mathrm{a}$ & $7,87 \mathrm{a}$ & $0,26 \mathrm{a}$ \\
\hline Enxertado & $0,43 \mathrm{a}$ & $0,07 \mathrm{~b}$ & $0,63 \mathrm{~b}$ & $3,22 \mathrm{c}$ & $0,26 \mathrm{a}$ \\
\hline \multicolumn{6}{|l|}{ Paraíso MG H 419-1 } \\
\hline Pé franco & $0,40 \mathrm{~b}$ & $0,08 \mathrm{ab}$ & $0,58 \mathrm{a}$ & $3,46 \mathrm{a}$ & $0,19 \mathrm{~b}$ \\
\hline Autoenxertado & $0,38 \mathrm{~b}$ & $0,06 \mathrm{~b}$ & $0,65 \mathrm{a}$ & $3,40 \mathrm{a}$ & $0,18 \mathrm{~b}$ \\
\hline Enxertado & $0,49 \mathrm{a}$ & $0,10 \mathrm{a}$ & $0,65 \mathrm{a}$ & $4,69 \mathrm{a}$ & $0,26 \mathrm{a}$ \\
\hline $\mathrm{CV}(\%)$ & 10,62 & 16,92 & 19,30 & 19,27 & 16,85 \\
\hline
\end{tabular}

Médias seguidas pela mesma letra na vertical - dentro de cada cultivar - não diferem entre si a 5\% ( $<<0,05)$ pelo teste de Tukey. 
enxertia e dos cultivares de $C$. arabica $\mathrm{L}$. utilizados. Não houve diferença significativa entre os tratamentos Apoatã autoenxertado e Apoatã pé franco, demonstrando que o Apoatã IAC 2258 pode ser utilizado como porta-enxerto, não sendo a enxertia prejudicial ao seu desenvolvimento (Tabela 4).

Na comparação entre Apoatã autoenxertado e os tratamentos em que as mudas eram enxertadas sobre o Apoatã, foi possível analisar se a copa de arábica influencia o desempenho do porta-enxerto. Pelos resultados obtidos, verifica-se que não houve influência de nenhum cultivar utilizado, como copa, na eficiência de uso dos nutrientes. Os resultados coincidem em parte com os apresentados por Fahl et al. (1998), que não encontraram dife- renças nos teores foliares de plantas enxertadas em Apoatã IAC 2258, ou não, com exceção do micronutriente manganês, pois as mudas enxertadas no Apoatã apresentaram menor índice de eficiência de uso, provavelmente pela maior seletividade do sistema radicular do Apoatã em relação ao manganês.

Tomaz et al. (2006) estudaram a translocação de nutrientes em quatro cultivares de Coffea arabica, enxertados, ou não, em Apoatã IAC 2258, Conilon Muriaé 1, Mundo Novo e RC EMCAPA 8141. Os autores concluíram que houve variações na absorção, translocação e utilização de $\mathrm{Zn}, \mathrm{Cu}$ e Mn nas mudas de cafeeiro, quando se compararam as combinações de enxertias com os respectivos pés francos.

Tabela 4. Eficiência de uso de boro (EUB), de ferro (EUFe), de zinco (EUZn), de cobre (EUCu) e de manganês (EUMn) em mudas de cafeeiro enxertadas, em relação ao Apoatã autoenxertado

\begin{tabular}{|c|c|c|c|c|c|}
\hline Contraste & $\begin{array}{c}\text { EUB } \\
\left(\mathrm{g}^{2} \cdot \mathrm{mg}^{-1}\right)\end{array}$ & $\begin{array}{l}\text { EUFe } \\
\left(\mathbf{g}^{2} \cdot \mathrm{mg}^{-1}\right)\end{array}$ & $\begin{array}{l}\text { EUZn } \\
\left(\mathbf{g}^{2} \cdot \mathbf{m g}^{-1}\right)\end{array}$ & $\begin{array}{l}\text { EUCu } \\
\left(\mathrm{g}^{2} \cdot \mathrm{mg}^{-1}\right)\end{array}$ & $\begin{array}{c}\text { EUMn } \\
\left(\mathrm{g}^{2} \cdot \mathrm{mg}^{-1}\right)\end{array}$ \\
\hline Apoatã autoenxertado & 0,44 & 0,08 & 0,72 & 3,61 & 0,28 \\
\hline vs apoatã pé franco & $0,44^{\mathrm{ns}}$ & $0,10^{\mathrm{ns}}$ & 0,69 ns & $4,73^{\mathrm{ns}}$ & $0,22 \mathrm{~ns}$ \\
\hline Apoatã autoenxertado & 0,44 & 0,08 & 0,72 & 3,61 & 0,28 \\
\hline vs Palma II enxertado & $0,54^{\mathrm{ns}}$ & $0,13^{\mathrm{ns}}$ & $0,94^{\mathrm{ns}}$ & $5,50^{\mathrm{ns}}$ & $0,30^{\mathrm{ns}}$ \\
\hline vs Catucaí 2SL enxertado & $0,36^{\mathrm{ns}}$ & $0,07^{\mathrm{ns}}$ & $0,56^{\mathrm{ns}}$ & $2,89^{\mathrm{ns}}$ & $0,23^{\mathrm{ns}}$ \\
\hline vs Oeiras enxertado & $0,54^{\mathrm{ns}}$ & $0,12^{\mathrm{ns}}$ & 0,70 ns & $5,01 \mathrm{~ns}$ & $0,32^{\mathrm{ns}}$ \\
\hline vs Obatã enxertado & $0,46^{\mathrm{ns}}$ & $0,09^{\text {ns }}$ & $0,71^{\mathrm{ns}}$ & $4,99^{\text {ns }}$ & $0,25^{\mathrm{ns}}$ \\
\hline vs Acauã enxertado & $0,48^{\mathrm{ns}}$ & $0,11^{\mathrm{ns}}$ & $0,63^{\mathrm{ns}}$ & $5,04^{\mathrm{ns}}$ & $0,22^{\mathrm{ns}}$ \\
\hline vs Topázio enxertado & 0,34 ns & $0,07^{\mathrm{ns}}$ & 0,63 ns & 3,22 ns & $0,26^{\text {ns }}$ \\
\hline vs Paraíso enxertado & $0,49^{\text {ns }}$ & $0,09^{\mathrm{ns}}$ & $0,65^{\text {ns }}$ & $4,68^{\mathrm{ns}}$ & $0,26^{\mathrm{ns}}$ \\
\hline
\end{tabular}

* e ns; contrastes significativos e não significativos, respectivamente, pelo teste de Dunnett a $5 \%(\mathrm{p}<0,05)$.

\section{CONCLUSÕES}

O porta-enxerto utilizado influenciou negativamente na absorção de boro, ferro e manganês.

A translocação dos micronutrientes boro e cobre obteve maiores índices nas mudas enxertadas.

O cultivar Palma II foi o que apresentou melhor eficiência de uso dos nutrientes quando enxertado, mostrando-se passível de ser enxertado.

O cultivar Apoatã IAC 2258 não sofreu influência negativa, tanto da técnica da enxertia, quanto dos cultivares utilizados, mostrando que pode ser utilizado como portaenxerto sem nenhum prejuízo ao seu desenvolvimento.

\section{AGRADECIMENTOS}

Ao Conselho Nacional de Desenvolvimento Científico e Tecnológico (CNPq) pela concessão da bolsa de PNPD (JCRA), à Coordenação de Aperfeiçoamento de Pessoal de Nível Superior (CAPES) pela concessão da bolsa de PNPD (AMC), ao Instituto Nacional de Ciência e Tecnologia do
Café (INCT - Café), ao Consórcio Pesquisa Café e à Fundação de Amparo à Pesquisa do Estado de Minas Gerais (FAPEMIG), pelo suporte financeiro ao projeto.

\section{REFERÊNCIAS}

Augusto HS, Martinez HEP, Sampaio NF, Cruz CD \& Pedrosa AW (2007) Concentração foliar de nutrientes em cultivares de Coffea arabica L. sob espaçamentos adensados. Ciência e Agrotecnologia, 31:973-981.

Caldeira MVW, Rondon Neto RM \& Schumacher MV (2004) Eficiência do uso de micronutrientes e sódio em três procedências de acácia-negra (Acacia mearnsii De Wild.). Revista Árvore, 28:39-47.

Favarin JL, Vitti GC, Dourado Neto D, Favarin Junior JL \& Salgado PR (2007) Teor de zinco no café como variável da disponibilidade no solo e extrator. Revista Brasileira de Ciência do Solo, 31:1191-1196.

Fahl JI, Carreli MLC, Gallo PB, Da Costa WM \& Novo M do C de SS (1998) Enxertia de Coffea arabica sobre progênies de Coffea canephora e de $C$. congensis no crescimento, nutrição mineral e produção. Bragantia, 57:297-312.

Ferreira DF (2008) SISVAR: um programa para análises e ensino de estatística. Revista Symposium, 6:36-41. 
Ferreira AD, Carvalho AM, Mendes ANG, Carvalho GR, Botelho CE \& Carvalho JG (2010) Absorção, translocação e eficiência no uso dos macronutrientes em cafeeiros (Coffea arabica) enxertados em Apoatã IAC 2258 (Coffea canephora). Interciencia, $35: 1-5$.

Gerloff GC \& Gabelman WH (1983) Genetic basis of inorganic plant nutrition. In: Laüchli A \& Bieleski RL (Eds.) Inorganic plant nutrition. Encyclopedia of Plant Physiology. Berlim, New York, Tokyo: Springer-Verlag. p.453-486.

Hoagland D \& Arnon DI (1950) The water culture method for growing plants without soil. California Agriculture Experimental Station Circular. Berkeley, California Agriculture Experimental Station. 347p.

Lange A, Martines AM, Silva MAC da, Sorreano MCM, Cabral CP \& Malavolta E (2005) Micronutrient deficiency effect on the nutritional status of the castor bean cultivar Iris. Pesquisa Agropecuária Brasileira, 40:61-67.

Li B, McKeand SE \& Allen HL (1991) Genetic variation in nitrogen use efficiency of loblolly pine seedlings. Forest Science, 37:613626

Marschner H (1995) Mineral nutrition of higher plants. San Diego, Academic Press. 889p.

Mattiello EM, Pereira MG, Zonta E, Mauri J, Matiello JD, Meireles PG \& Silva IR da (2008) Produção de matéria seca, crescimento radicular e absorção de cálcio, fósforo e alumínio por Coffea canephora e Coffea arabica sob influência da atividade do alumínio em solução. Revista Brasileira de Ciência do Solo, 32:425434.

Natale W, Prado R de M, Leal RM \& Franco CF (2004) Efeitos da aplicação de zinco no desenvolvimento, no estado nutricional e na produção de matéria seca de mudas de maracujazeiro. Revista Brasileira de Fruticultura, 26:310-314.
Siddiqi MY \& Glass ADM (1981) Utilization index: a modified approach to the estimation and comparison of nutrient utilization efficiency in plants. Journal of Plant Nutrition, 4:289302.

Swiader JM, Chyan Y \& Freiji FG (1994) Genotypic differences in nitrate uptake and utilizatin efficiency in pumpkin kibrids. Journal of Plant Nutrition, 17:310-314.

Soares CRFS, Grazziotti PH, Siqueira JO, Carvalho JG de \& Moreira FMS (2001) Toxidez de zinco no crescimento e nutrição de Eucalyptus maculata e Eucalyptus urophylla em solução nutritiva. Pesquisa Agropecuária Brasileira, 36:339-348.

Tomaz MA, Silva SR, Sakiyama NS \& Martinez HEP (2003) Eficiência de absorção, translocação e uso de cálcio, magnésio e enxofre por mudas enxertadas de Coffea arabica. Revista Brasileira de Ciência do Solo, 27:885-892.

Tomaz MA, Martinez HEP, Sakiyama NS, Cruz CD \& Pereira AA (2006) Absorção, translocação e utilização de zinco, cobre e manganês por mudas enxertadas de Coffea arábica. Revista Brasileira de Ciência do Solo, 30:377-384.

Tomaz MA, Martinez HEP, Cruz CD, Ferrari RB, Zambolim L \& Sakiyama NS (2008) Diferenças genéticas na eficiência de absorção, na translocação e na utilização de $\mathrm{K}, \mathrm{Ca}$ e $\mathrm{Mg}$ em mudas enxertadas de cafeeiro. Ciência Rural, 38:1540-1546.

Willson KC (1987) Mineral nutrition and fertilizer needs. In: Clifford MMN \& Willson KC (Eds.) Coffe: botany, biochemistry and productions of beans and beverage. London, Croom Helm. $156 \mathrm{p}$. 